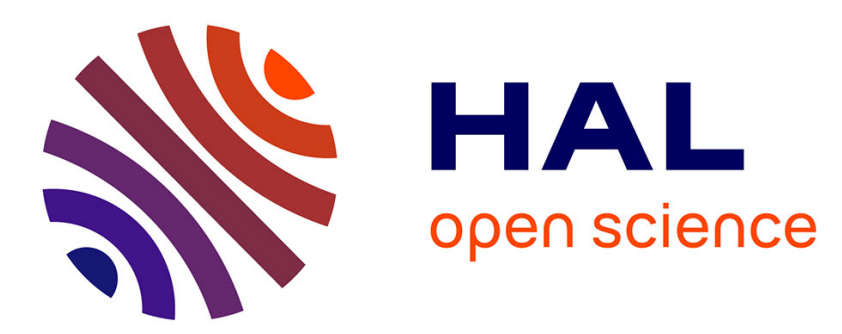

\title{
Dissolution d'un groupe ethnique. Origines des témoins et des conjoints des enfants des familles polonaises implantées dans le Cher 1940-1975
}

\author{
Philippe Rygiel
}

\section{- To cite this version:}

Philippe Rygiel. Dissolution d'un groupe ethnique. Origines des témoins et des conjoints des enfants des familles polonaises implantées dans le Cher 1940-1975. Le Mouvement social, 2000, 191, pp.69-89. halshs-00383357

\section{HAL Id: halshs-00383357 \\ https://shs.hal.science/halshs-00383357}

Submitted on 13 May 2009

HAL is a multi-disciplinary open access archive for the deposit and dissemination of scientific research documents, whether they are published or not. The documents may come from teaching and research institutions in France or abroad, or from public or private research centers.
L'archive ouverte pluridisciplinaire HAL, est destinée au dépôt et à la diffusion de documents scientifiques de niveau recherche, publiés ou non, émanant des établissements d'enseignement et de recherche français ou étrangers, des laboratoires publics ou privés. 
Dissolution d'un groupe ethnique.

Origines des témoins et des conjoints des enfants des familles polonaises implantées dans le Cher 1940-1975. Par Philippe Rygiel ${ }^{1}$.

Mouvement Social. Numéro 191, avri1-juin 2000

L'histoire de l'immigration a rarement, en France, étudié de manière systématique ce que la tradition sociologique nomme l'assimilation structurelle ${ }^{2}$ des populations issues de l'immigration. Les auteurs des travaux consacrés à l'intégration des populations issues de l'immigration, considérée par eux comme un cas particulier de l'assimilation nationale des populations de la France ${ }^{3}$, se sont surtout intéressés aux institutions permettant celle-ci ${ }^{4}$, ou aux mécanismes conduisant à une assimilation souvent conçue, ou plutôt étudiée, comme la résultante de l'action et du fonctionnement d'un État moderne, dans sa double dimension d'État-Nation et d'État social ${ }^{5}$, et entendue comme l'incorporation par les populations issues de l'immigration des normes politiques et culturelles régissant la société française et l'abandon, ou le refoulement vers le privé, des valeurs, symboles et pratiques dénotant ou dénonçant l'origine ${ }^{6}$. A suivre ces textes, lorsque les mécanismes d'assimilation fonctionnent, cette acculturation s'accompagne chez les sujets d'une identification à la nation française et d'un rejet/refoulement des autres identités

\footnotetext{
${ }^{1}$ Professeur d'histoire-géographie au Lycée technique Pierre-Emile-Martin de Bourges.

2 GORDON Milton M., "Assimilation in America: Theory and Reality", Daedalus, 90, Spring 1961.

3 Dominique Schnapper écrit: "Si l'on accepte ces analyses on admettra que la présence de populations d'origine immigrée (...) ne fait que traduire ou occulter le véritable problème, celui de l'intégration nationale." in SCHNAPPER D., La France de l'intégration. Sociologie de la nation en 1990, Paris, Gallimard, 1991, page 103. Gérard Noiriel lui écrit : "Nous allons montrer dans les pages qui suivent que ce sont les mêmes facteurs qui ont permis à la fois l'assimilation des diverses composantes régionales de la population française dans le passé et l'intégration des millions d'immigrés installés en France dans la période récente." in NOIRIEL G., Population, immigration et identité nationale en France XIX ${ }^{e}-X X^{e}$ siècle, Paris, Hachette, 1992, page 86.

${ }^{4}$ SCHNAPPER D., La France de l'intégration, opus cité.

5 BLANC-CHALEARD M.C. (dir.) ," Immigration et logiques nationales. Europe, XIX ${ }^{\mathrm{e}}-\mathrm{XX}^{\mathrm{e}}$ siècle", Le Mouvement Social, numéro 188, 1999.

${ }^{6}$ Nous trouvons une formulation lapidaire de cette idée chez Dominique Schnapper et Rémy Leveau lorsqu'ils évoquent les enfants des migrants de l'entre-deux-guerres : " Si la génération des migrants maintint l'essentiel de la vie privée de leur pays d'origine jusqu'à la guerre, il n'en alla pas de même avec leurs enfants. Les témoignages sont unanimes pour constater que les enfants de ces migrants restés plus ou moins étrangers au pays dans lequel ils avaient dû s'installer, sont devenus français non seulement par la nationalité, mais par la culture, même si certains continuaient d'entretenir des liens sentimentaux avec le pays d'origine de leurs parents" in LEVEAU R., SCHNAPPER D., "Etre immigré en France", in ARIES P, DUBY G.(dir.), Histoire de la vie privée, Tome 5, Paris, Seuil, 1987, page 508.
} 
nationales possibles, particulièrement lorsque celles-ci font l'objet d'un stigmate ${ }^{7}$. De la définition d'un tel programme de recherche par les auteurs faisant référence dans le champ, de la méfiance persistante envers les travaux qui pourraient conduire à faire de l'origine nationale, ou ethnique, une variable déterminante des comportements, du fait aussi que l'assimilation des populations issues des immigrations anciennes est souvent postulée, de l'absence, enfin, de données agrégées au niveau national ${ }^{8}$, qui contraint à recourir à l'approche monographique, difficile en l'occurrence à mettre en oeuvre, vient que nous savons peu de choses de la composition des réseaux familiaux et des cercles de sociabilité que les descendants des immigrants installés en France rejoignirent. Nos connaissances sont d'autant plus lacunaires que la vague migratoire qui emporta leurs parents est ancienne. Aucun des auteurs du volume consacré par la Revue du Nord aux membres de la Polonia présents en France après $1945^{9}$ n'aborde ni ne mentionne ce thème et nous ne disposons que de quelques études, vieilles souvent de plusieurs décennies, qui soient consacrées aux comportements matrimoniaux ou à la sociabilité des enfants des migrants polonais arrivés en France durant l'entre-deux-guerres ${ }^{10}$. La question est cependant d'importance. D'une part, les historiens s'interrogent aujourd'hui sur les facteurs permettant, ou empêchant, l'apparition, au sein des vieux États-Nations, de "communautés imaginés", que l'on dit issues de l'immigration et qui constitueraient des entités sociohistoriques nouvelles ${ }^{11}$. Il nous faut donc réexaminer les formes de l'assimilation des enfants des anciennes immigrations et nous prononcer sur leurs éventuelles spécificités. D'autre part, s'affirme la tentation, dans certains milieux scientifiques, de rendre compte des pratiques et des choix identitaires des enfants

7 Gérard Noiriel note ainsi que : "(..) l'un des principaux critères de définition de la "deuxième génération" immigrée tient à sa volonté de s'intégrer dans la société qui est la sienne; mais que les obstacles rencontrés provoquent un rejet plus ou moins marqué de tout ce qui rappelle les origines." in NOIRIEL G. Le creuset français, histoire de l'immigration (XIX $X^{\circ} X X^{\circ}$ siècle), Paris, Le Seuil, 1988, page 234.

8 MUNOZ-PEREZ F., "Observation statistique des mariages mixtes", Hommes et Migrations, numéro 1167, 1993.

9 GOGOLEWSKI E., "Les ouvriers polonais en France après la seconde guerre mondiale", Revue du Nord, hors série numéro 7, Université Charles de Gaulle, Lille III, 1992.

10 GIRARD A., STOETZEL J., Français et immigrés, Paris, Puf/Ined, Travaux et documents, Cahier numéro 19, 1953; POIGNANT R., "Étude sur l'assimilation de l'immigration polonaise dans le Pas de Calais, Population, IV-1, Janvier-Mars 1949. STANIEC J., Intégration et ascension sociale des Polonais du Pas-de-Calais depuis 1945, Mémoire de DEA sous la direction de Janine Ponty, Besançon, Université de Franche-Comté, 1991.

11 BLANC-CHALEARD M.C., "Des logiques nationales aux logiques ethniques", Mouvement Social, numéro 188, Juillet-Septembre 1999. 
d'immigrés en se référant exclusivement à la culture ou à l'origine de leurs parents ${ }^{12}$, ce qui s'accompagne souvent de la conviction que l'existence de groupes "socio-ethniques" rassemblant les enfants des immigrés de même origine est de l'ordre de l'évidence ${ }^{13}$ et que cela suffit à justifier l'introduction des "catégories "ethniques" dans l'appareil statistique d'état ${ }^{14}$." Le mariage des enfants des immigrés devient alors le "point central du processus d'assimilation 15 ", ou du moins des débats traitant de celui-ci, puisque l'union de deux jeunes gens de même origine peut être compris, à la fois comme l'expression du poids de la culture des parents sur le destin et les choix des enfants ${ }^{16}$ et comme le révélateur de leurs choix identitaires, voire comme le signe de l'évidence de l'existence du groupe défini par une commune origine en tant que groupe socioethnique $^{17}$. L'étude des mariages des enfants des immigrés polonais installés dans le département du Cher avant 1945 nous permet de contribuer à ces débats. Nous pouvons, la menant, préciser notre connaissance des formes et des déterminants de l'assimilation sociale d'une population issue d'une immigration ancienne, tout en nous interrogeant sur la valeur heuristique de l'appel à la culture ou à l'origine des parents, lorsqu'il s'agit de rendre compte des pratiques et des choix des enfants d'immigrés, voire nous demander si la simple observation, au niveau local, d'une forte endogamie, suffit à prouver la pertinence d'un classement social distinguant des groupes dont les membres partagent une même origine. Cela permet alors de s'interroger sur la valeur de quelquesuns des arguments mobilisés par les tenants des statistiques ethniques.

\section{Lieux et outils de la recherche}

Ce travail s'appuie sur l'exploitation de données collectées lors de la préparation d'une thèse consacrée à la mobilité sociale et géographique des familles immigrées s'étant installées dans le Cher entre 1920 et $1945^{18}$. Nous avions alors relevé les renseignements

12 Pour une étude, et non un exemple, de ces discours voir LIAUZU C., "L'obsession des origines : démographie et histoire des migrations", Mots/Les langages du politique, numéro 60, septembre 1999.

13 TRIBALAT M., "De la nécessité de reformuler la question de l'immigration en France, DUPAQUIER J., Morales et politiques de l'immigration, PUF, Paris, 1998, pages 4576.

14 MERLLIE D., SPIRRE A., "La question des origines dans les statistiques en France. Les enjeux d'une controverse", Le mouvement social, juillet-septembre 1999, numéro 188, page 119 .

15 TRIBALAT M., De l'immigration à l'assimilation, La Découverte/Ined, Paris, 1996, page 263 .

16 idem, pages 263-264.

17 CHIGNIER-RIBOULON F., L'intégration des Franco-Maghrébins. L'exemple de l'est lyonnais, L'Harmattan, Paris, 1999.

18 RYGIEL P., Mais où sont les immigrés d'antan? Trajectoires sociogéographiques de familles issues de l'immigration européenne implantées dans le Cher durant l'entre- 
portés sur les actes de naissance de la quasi totalité des enfants, nés dans le département entre 1923 et 1945, dont l'un au moins des parents était né en Pologne ou en Allemagne et portait un patronyme polonais. La présence, en marge de l'acte de naissance, du lieu et de la date du mariage de ceux-ci, nous avait permis de retrouver l'acte dressé lors de leur premier mariage, lorsqu'ils s'étaient mariés dans le Cher, dans l'un des départements limitrophes à celui-ci ou à Paris et de saisir les renseignements que portait ce document. La comparaison de la population ainsi constituée et de celle que permettaient d'étudier les fichiers du personnel des principaux employeurs de main d'oeuvre étrangère de la région et les listes nominatives des recensements nous conduisait à conclure que cette base de données ne nous autorisait pas à étudier une vague migratoire, mais nous plaçait dans des conditions acceptables dès lors qu'il s'agissait d'examiner les sédiments déposés par celle-ci ${ }^{19}$ : nous pouvions décrire le parcours sociogéographique de la majorité des enfants des familles originaires de Pologne dont le séjour dans notre département avait duré plus que quelques années.

L'inscription, en marge de l'acte de naissance, du nom et du prénom du conjoint, celle, sur l'acte de mariage, des noms et prénoms des témoins présents, nous offre de plus des indices relatifs à l'origine des participants à cette cérémonie. Le port d'un patronyme polonais ${ }^{20}$ ne saurait certes constituer la preuve qu'un conjoint est lui même polonais, ou enfant de polonais, non plus d'ailleurs que ne pas posséder un tel nom signifie nécessairement que l'on ne soit pas d'origine polonaise. Nous ne pouvons donc être certains de l'origine d'aucun des conjoints et des témoins étudiés. Les particularités de l'immigration polonaise de l'entre-deux-guerres sont telles, cependant, que les risques d'erreur sont assez limités. L'entrée en France des immigrés polonais se déroule durant une période très courte. Très rares avant la première guerre mondiale, les arrivées de Polonais en France le sont tout autant après le second conflit mondial ${ }^{21}$. Il y a de ce fait peu de chances que les enfants d'ouvriers polonais que nous étudions épousent des descendants d'une immigration polonaise plus ancienne, dont seul leur nom garderait le souvenir. De plus, les Polonais et leurs enfants sont peu nombreux à faire franciser leur nom. Notons, à titre d'exemple, que seuls 7 des 1268 enfants nés d'un père polonais présents dans notre base de données obtinrent, d'après les registres d'État-civil, que leur

deux-guerres, Thèse d'Histoire sous la direction de Janine Ponty, Université de FrancheComté, 1996. Une version revue et abrégée de cette étude sera publiée cette année par les Presses de l'Université de Franche-Comté.

${ }^{19}$ RYGIEL P., Mais où sont ..., opus cité, pages 92-98.

${ }^{20}$ Une précédente enquête permet d'estimer que la proportion de noms polonais non identifiés comme tels est inférieure à $6 \%$. RYGIEL., Mais où sont ..., opus cité, pages 80-81. La probabilité de considérer polonais un nom patronymique ne l'étant pas est par contre inconnue.

${ }^{21}$ PONTY J., Polonais méconmus. Histoire des travailleurs immigrés en France dans l'entre-deux-guerres, Paris, Publications de la Sorbonne, 1988. 
nom patronymique soit modifié. Ajoutons que les mariages mixtes unissant une Polonaise et un Français sont extrêmement rares durant l'entre-deux-guerres ${ }^{22}$, soit pendant la période durant laquelle naissent les futurs conjoints des enfants de Polonais étudiés. Enfin, il ne se trouve parmi les populations présentes localement aucun groupe dont les membres posséderaient des noms patronymiques assez semblables aux noms polonais pour que de nombreuses confusions soient possibles, alors qu'une étude du même ordre, menée sur une population d'origine italienne, serait condamnée à l'échec, du fait de la présence de nombreux Corses au sein des villes du Cher. Nous pouvons de ce fait estimer que la méthode utilisée produit des erreurs peu nombreuses et nous permet de disposer d'estimations grossières. $\mathrm{Si}$ en outre nous postulons ces erreurs aléatoirement réparties, ce que n'interdit aucun des éléments en notre possession, nous sommes en mesure de supposer que ces erreurs n'altèrent pas notre perception des phénomènes étudiés

Les données ainsi constituées sont cependant incertaines, grossières, et produites au moyen d'un dispositif difficilement reproductible, ce qui nous obligera à ne considérer les résultats obtenus que comme des ordres de grandeur et des indices.

Ces limites posées, nos sources permettent de se prononcer sur l'origine du conjoint de 772 enfants nés entre 1923 et 1945 d'au moins un parent polonais. La trajectoire sociogéographique de 532 d'entre eux est connue par leur acte de mariage, qui nous permet également de formuler des hypothèses quant à l'origine des témoins assistant à cette cérémonie. Cette population regroupe des individus provenant de milieux géographiques et professionnels divers. Un peu moins du tiers d'entre eux sont les enfants des ouvriers métallurgistes de l'usine de seconde fusion de Rosières. Celle-ci, installée au creux d'une boucle du Cher, à l'écart de toute agglomération importante, est une "usine à la campagne ${ }^{23 "}$ ", qui eut massivement recours durant les années vingt à une main d'oeuvre polonaise affectée aux travaux pénibles et dangereux de la fonderie. Logés dans la cité accolée à l'usine, nombre de ces travailleurs ont fait venir leur famille et sont parvenus à constituer, à l'ombre d'un paternalisme vigoureux, une petite communauté polonaise que structurent ses associations et ses fêtes ${ }^{24}$. Leur situation diffère sensiblement de celle des enfants d'ouvriers agricoles et de cultivateurs, qui constituent plus du tiers de notre population, dont les parents, à moins de travailler pour l'exploitation betteravière de Laverdines, qui emploie en permanence quelques dizaines de travailleurs polonais et tchécoslovaques, sont dispersés dans les fermes de la

${ }^{22}$ MUNOZ-PEREZ F., TRIBALAT M., "Mariages d'étrangers et mariages mixtes en France. Évolution depuis la première guerre mondiale", Population, XXXIX-3, Mai-Juin 1984.

23 PIGENET M., "Rosières, l'usine et le village", Le Mouvement social numéro 119 , avril-juin 1982

24 RYGIEL P., "La formation de la colonie polonaise de Rosières", Cahiers d'archéologie et d'histoire du Berry, numéro 129, mars 1997. 
Champagne berrichonne et entretiennent rarement de contacts quotidiens avec d'autres immigrés polonais. Proviennent aussi de ces campagnes agricoles la vingtaine d'enfants nés d'un père né en France et d'une mère polonaise qu'abrite notre fichier. Le reste de notre population est plus disparate. Nous y trouvons les enfants des ouvriers polonais présents durant l'entre-deux-guerres dans les grandes villes du département et les campagnes industrialisées de l'est et du sud de celui-ci, parsemées de grosses bourgades et de petites villes, groupées souvent, telles Grossouvre ou Beffes, autour d'une unique usine. Peu nombreux à disposer d'une qualification monnayable sur le marché du travail local - seuls $6 \%$ des membres de notre population sont, d'après la profession déclarée par leur père lors de leur naissance, fils d'ouvrier qualifié - leurs pères s'embauchent dans une entreprise qui emploie avant la seconde guerre mondiale un petit noyau d'ouvriers polonais, telle l'usine de ciment Poliet et Chausson de Beffes, la tôlerie Labbé à SaintFlorent-sur-Cher ou la tuilerie Lavalée de Grossouvre, près de Sancoins, ou bien se perdent dans la ville; quelques dizaines travaillent ainsi pour des verreries ou des usines de porcelaine de la région de Vierzon. C'est généralement au sein de ces populations que l'on rencontre les quelques immigrants qui, s'unissant avec une femme née en France, ou une autre étrangère, donnent naissance aux rares enfants de couples mixtes que compte notre fichier.

\section{Rareté des mariages entre enfants de Polonais}

Les enfants des Polonais du Cher contractent rarement mariage avec un ou une conjointe possédant un patronyme polonais. Ce n'est le cas que de $20 \%$ d'entre eux. Nous sommes loin des taux obtenus par Alain Girard et Jean Stoetzel lorsqu'ils observaient les mariages des fils et des filles des ouvriers agricoles de l'Aisne ou des mineurs du Nord-Pas-de-Calais, puisque seuls 55\% des premiers et $25 \%$ des seconds contractaient un mariage mixte ${ }^{25}$. De même, les trois quarts des enfants de Polonais du Pas-de-Calais évoqués par Raymond Poignant ont contracté mariage avec un individu d'origine polonaise ${ }^{26}$.

Plusieurs éléments peuvent expliquer ces écarts. D'une part les dispositifs d'enquête et les catégories utilisés par ces études ne sont pas identiques aux nôtres. Elles s'appuient sur des entretiens ou l'exploitation de questionnaires et l'on peut supposer que peu de mariages contractés avec des individus d'origine polonaise leur échappent, alors que nous sommes amenés à légèrement sous-estimer le nombre de ceux-ci, du fait de la nature de nos données. L'écart cependant est tel qu'il est douteux que cela puisse l'expliquer. Les particularités des populations étudiées y contribuent. Nous étudions des

${ }^{25}$ GIRARD A., STOETZEL J., Français et immigrés, opus cité, page 81.

${ }^{26}$ POIGNANT R., "Étude sur l'assimilation de l'immigration polonaise ...", opus cité. 
individus plus jeunes, et se mariant plus tardivement, que les auteurs de ces travaux. L'ouvrage de Girard et Stoetzel est publié en 1953, l'article de Poignant en 1949, et il ne prend en compte que les mariages d'enfants de travailleurs polonais antérieurs à 1948 . La plupart d'entre eux sont nés dans les années vingts, certains étant sans doute nés en Pologne et y ayant grandi. A l'inverse, nous étudions le parcours d'enfants nés, donc socialisés, en France, dont nous savons que les pratiques diffèrent considérablement de celles de leurs aînés venus en France dans l'enfance et l'adolescence, qui sont, quel que soit le courant migratoire et l'époque, beaucoup plus nombreux à épouser un conjoint dont ils partagent l'origine que leurs cadets ${ }^{27}$. De plus, les couples que nous étudions se sont formés entre 1940 et 1988, 92\% des fils présents convolant entre 1948 et 1971 et 94\% des filles se mariant entre 1946 et 1971. De ce fait, nous examinons des unions presque toutes contractées postérieurement à la période étudiée par les auteurs cités, qui de plus unissent des individus plus jeunes, puisque la majorité de nos sujets sont nés après 1930. Or, tant la période durant laquelle se déroule le mariage, que la génération à laquelle appartiennent les mariés, influent sur la propension à contracter mariage avec un conjoint de même origine (cf. graphique ci-dessous ${ }^{28}$ ).

\section{Des cohortes aux destins dissemblables}
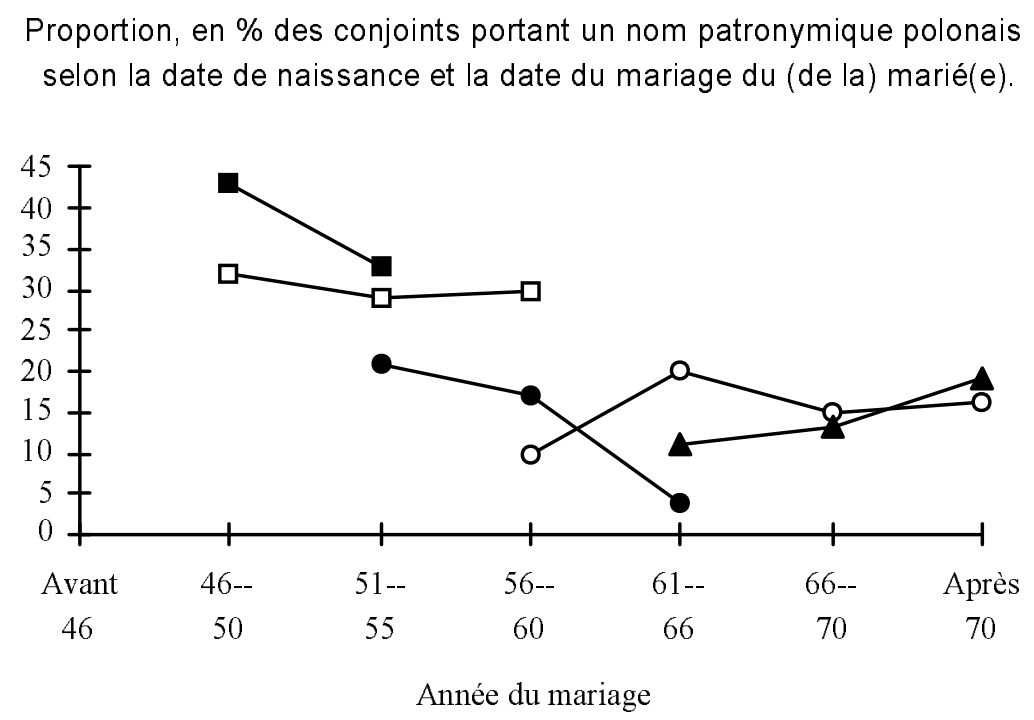

Année de naissance

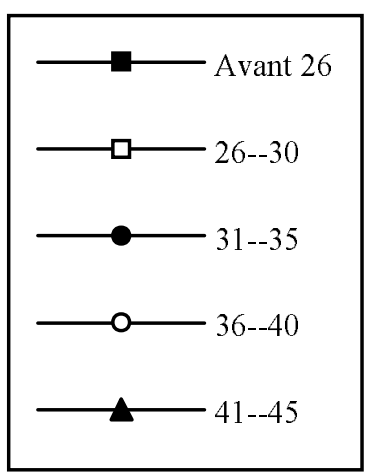

Ce graphique permet de représenter simultanément les effets de cohorte, d'âge et de période. Les enfants de Polonais du Cher se mariant durant la même période ont d'autant plus de chances d'épouser un conjoint de même origine qu'eux qu'ils sont nés

27 TRIBALAT M., Faire France, Paris, La Découverte, pages 68-69.

${ }_{28} \mathrm{Ne}$ sont portés sur le graphique ci dessous que les valeurs obtenues à l'aide de l'observation d'au moins dix mariages. 
tôt. Cependant, la diminution de la propension à épouser un conjoint de même origine n'est pas régulière. Ce graphe permet d'opposer les enfants nés avant 1931, regroupés dans le haut de la figure, dont un tiers environ épousent un partenaire d'origine polonaise, et leurs cadets, qui ne sont que de 10 à $20 \%$ à le faire.

S'il semble que, à même année de naissance, les enfants de Polonais soient d'autant plus enclins à épouser un partenaire d'une autre origine que la leur que le mariage se produit plus tard, ce que traduit l'orientation vers le bas de quatre de ces cinq courbes, l'influence de ce facteur semble moindre que celle du précédent. La pente de ces courbes est généralement faible et irrégulière. L'agrégation des données utilisée ci-dessus, qui permet de disposer d'effectifs plus étoffés, tout en éliminant l'effet de la variable sexe, sensible ici puisque les filles tendant à se marier avant les garçons, confirme le faible poids de cette variable.

Conjoints ayant un patronyme d'origine polonaise, selon l'année de naissance et la date du mariage.

\begin{tabular}{|c|c|c|c|c|c|c|c|c|c|c|c|c|}
\hline Mariage & \multicolumn{4}{|c|}{ Précoce } & \multicolumn{4}{|c|}{ Tardif } & \multicolumn{4}{|c|}{ Total } \\
\hline Année de naissance/Patronyme & \multicolumn{2}{|c|}{ Polonais } & \multicolumn{2}{|c|}{ Non polonais } & \multicolumn{2}{|c|}{ Polonais } & \multicolumn{2}{|c|}{ Non polonais } & \multicolumn{2}{|c|}{ Polonais } & \multicolumn{2}{|c|}{ Non polonais } \\
\hline & en $\%$ & Eff. & en $\%$ & Eff. & en $\%$ & Eff. & en $\%$ & Eff. & en $\%$ & Eff. & en $\%$ & Eff. \\
\hline Avant 1926 & 42,9 & 3 & 57,1 & 4 & 25 & 2 & 75 & 6 & 33 & 5 & 66,7 & 10 \\
\hline De 1926 à 1930 & 22 & 11 & 78 & 39 & 33,3 & 7 & 66,7 & 14 & 25 & 18 & 74.6 & 53 \\
\hline De 1931 à 1935 & 18,2 & 12 & 81,8 & 54 & 21,9 & 7 & 78,1 & 25 & 19 & 19 & 80.6 & 79 \\
\hline De 1941 à 1945 & 12,9 & 4 & 87,1 & 27 & 15,4 & 8 & 84,6 & 44 & 15 & 12 & 85,5 & 71 \\
\hline Total & 18,4 & 40 & 81,6 & 177 & 21,4 & 30 & 78,6 & 110 & 20 & 70 & 80,4 & 287 \\
\hline
\end{tabular}

Nous avons réparti les individus de sexe masculin nés durant une même période en deux sous-groupes, en fonction du moment de leur mariage, de façon à ce que les effectifs de chacun de ces deux sous-ensemble soient les plus similaires possibles, compte tenu des discrétisations selon la date du mariage déjà opérées. Nous pouvons alors, pour chaque groupe de personnes du même âge, comparer le destin des premiers mariés à celui de ceux de leurs contemporains qui se sont mariés plus tardivement qu'eux. Nous retrouvons les écarts selon l'année de naissance mis en lumière plus haut. Par contre, les variations selon la date du mariage apparaissent, pour une même génération, faibles, ne sont pas toujours de même sens, ni significatives, au regard des résultats des test statistiques usuels ${ }^{29}$, que l'on considère l'ensemble de la population observée, ou l'un quelconque de ces segments. Les écarts mis en évidence doivent donc être rapportés à un effet de cohorte et non à des effets d'âge, tandis que nous ne pouvons percevoir d'effets de période.

${ }^{29} \mathrm{Chi} 2$, test exact de Fisher. 
Des travaux précédents nous avaient permis de constater que le destin social des enfants de Polonais du Cher nés durant les années vingt différait sensiblement de celui de leurs cadets. Peu nombreux à poursuivre des études, ils entraient souvent très tôt sur le marché du travail et occupaient des postes d'ouvriers peu qualifiés, au contraire des plus jeunes dont une proportion notable parvenait à accéder au travail ouvrier qualifié, voire, particulièrement dans le cas des filles, aux emplois de bureau. Ces écarts dans les destins sociaux, autant que les facteurs qui les déterminent, contribuent à ce que les plus jeunes enfants des familles polonaises soient peu nombreux à épouser des partenaires partageant leur origine. Aussi reviendrons nous ici brièvement sur ce thème.

Les plus âgés des enfants d'immigrés que nous avons interrogés ont souvent quitté l'école dès la fin de leur scolarité obligatoire. C'est le cas de monsieur S., fils d'un manoeuvre polonais de Rosières, né en 1919, arrivé à Rosières en 1923, qui, juste après sa sortie de l'école, a "fait le vacher pendant quatre mois", avant d'être embauché par l'usine, ou de monsieur K., né en 1926, fils d'un manoeuvre polonais de Rosières, lui aussi "parti garder les vaches" dès sa scolarité achevée, avant de s'embaucher comme manoeuvre dans une entreprise de Saint-Florent-sur-Cher. Embauche rapide, que monsieur K. explique par la nécessité économique, il n'a pas poursuivi d'études parce que ses parents dit-il :

" (...) n'avaient pas les moyens. Et puis pour eux c'était le travail, le salaire. Quand j'ai commencé à travailler je rapportais le salaire à la maison. Ils étaient contents d'avoir ça pour payer les dettes."

A la nécessité s'ajoute la logique du projet migratoire des parents, qui a pour objectif l'accumulation d'un pécule permettant un retour rapide en Pologne et l'achat sur place d'une affaire ou de terres. Presque tous les enfants d'immigrés polonais rencontrés affirment en effet, telle Madame T., que leurs parents sont venus :

"en pensant rester deux ans, trois ans, se faire un pécule, acheter de la terre, pour pouvoir rentrer ${ }^{30}$."

Un tel projet n'a de chance d'être réalisé qu'au prix d'une mobilisation de toutes les énergies et de tout le temps familial. Les enfants participent très jeunes à cet effort, ce qui laisse peu de place au travail scolaire. Monsieur K. ${ }^{31}$, déjà cité, se souvient en ces termes de son enfance :

" J'allais au bois, ce n'est pas que ça me plaisait tellement (...) ou ils nous embauchaient à la ferme pour aller déterrer les betteraves, parce que quand ils prenaient

\footnotetext{
${ }^{30}$ Entretien $\mathrm{N}^{\circ} 9$, Mme T., fille de travailleurs polonais de Rosières.

${ }^{31}$ Il n'est pas le seul à évoquer de tels souvenirs Madame T., née en 1920 évoque les obligations qui pesaient sur les enfants en ces termes: "Il fallait aussi aider pour le jardin, ramasser des herbes pour les lapins; c'était important à l' époque parce qu'avec un salaire il était difficile de faire vivre une famille et d'élever quatre enfants, en plus ma mère était malade et mon père envoyait régulièrement de l'argent en Pologne à sa famille".
} 
un hectare de betterave à arracher il fallait les arracher rapidement, alors ils nous emmenaient. On avait 10 ou 11 ans, on peinait moins que nos parents à détasser. Ils nous faisaient aussi désherber dans les jardins, et puis il fallait qu'on garde les poules. Après les moissons on allait glaner, c'était ça de moins qu'ils avaient à acheter, et puis on ramassait de l'herbe pour les lapins, on savait lesquelles ramasser. Il y avait tout pour s'occuper, mes parents avaient des oies, et bien tous les jours après l'école il fallait que j'aille garder les oies. Puis je voyais les gamins qui jouaient au foot à côté. Moi je disais à mes parents : "Les Français ils ne vont pas au bois". Et puis ils n'élevaient pas de bêtes non plus."

Cependant, ce schéma se modifie après la guerre. D'une part à partir des années cinquante la condition ouvrière s'améliore indiscutablement et rapidement. La nécessité ne pèse plus si fortement sur les destins, ce qui permet d'accepter, si cela doit autoriser l'ascension sociale des enfants, les sacrifices financiers qu'exige une poursuite d'études. Beaucoup, de plus, prennent conscience, après les retours massifs de la fin des années quarante et la stabilisation du régime polonais, que le retour est une perspective bien lointaine, sinon illusoire. L'avenir, sinon des parents, du moins des enfants, est en France. La mobilisation intense du travail familial perd donc alors tant de sa nécessité que de sa légitimité, cependant que l'acquisition par les enfants d'un diplôme ou d'une qualification prend plus d'importance, d'autant sans doute que les aînés eux-mêmes peuvent, dans certains cas, se faire les avocats des plus jeunes. Madame T., ainsi, qui achève sa scolarité primaire à Rosières, avant de travailler en usine, a une soeur cadette qui a poursuivi des études qui en ont fait une institutrice, en partie, nous dit Madame T., parce que :

"mon mari et moi, on a fait comprendre à mes parents que c'était important.

Cette transformation des pratiques, qui coïncide avec les mutations de l'offre scolaire $^{32}$ et des structures du marché du travail ${ }^{33}$ qui caractérisent la France de l'expansion, permettra à beaucoup, parmi les plus jeunes des enfants étudiés, d'obtenir des qualifications monnayables et donc des emplois qualifiés, dont la poursuite, souvent, les entraînera loin des colonies polonaises de l'entre-deux-guerres.

Ces évolutions pèsent de plusieurs façons sur le choix du conjoint. D'une part, reproduisant moins souvent que leurs frères et soeurs la condition des parents, résidant moins souvent qu'eux en des lieux marqués par une forte présence immigrée ${ }^{34}$ - ce qui nous conduira à examiner les liens existant entre l'origine du partenaire et le parcours sociogéographique des enfants - les cadets ont accès à des lieux de rencontre, à des

32 PROST A., L'enseignement et l'éducation en France, Tome 4 : L'école et la famille dans une société en mutations, Paris, G.V. LABAT, 1981.

33 THEVENOT L., "Les catégories sociales en 1975, 1'extension du salariat", Économie et statistique, numéro 91, pages 23-24.

${ }^{34}$ RYGIEL P., Mais où sont, opus cité, pages 417-427. 
réseaux de sociabilité et à des marchés matrimoniaux sur lesquels se présentent une moindre proportion de partenaires d'origine polonaise.

De plus, l'adolescence des enfants nés durant les années trente se déroule alors que l'idée du retour au pays n'est plus un but orientant choix et pratiques quotidiennes, ce qui est susceptible de rendre plus acceptable par les familles le choix d'un partenaire qui les attachera définitivement à la France, choix qui ne remet plus en cause les projets familiaux. Nous pouvons également supposer que les plus jeunes des enfants de migrants étudiés ont, plus tôt et plus souvent que leurs aînés, considéré que ne pas épouser un partenaire d'origine polonaise était admissible, le retour étant devenu impossible, ce qui a pu orienter leurs pratiques et leurs choix. D'autre part, certains peuvent bénéficier, en cas de conflits familiaux, de l'appui de leurs aînés, dont nous avons vu qu'ils assument parfois le rôle de médiateur entre parents et cadets, ce que ne pouvaient espérer, du fait des caractéristiques démographiques de cette population, les enfants nés durant les années vingt, dont beaucoup sont des premiers nés.

Enfin, aînés et cadets ne comptent pas en leurs rangs la même proportion d'enfants de couples mixtes. Or les enfants qui n'ont qu'un parent d'origine polonaise ont beaucoup moins de chances que les autres membres de notre population d'épouser un partenaire de nom polonais. C'est le cas de 10\% des 81 enfants dont l'un des parents est né en France, mais de 7\% des 29 personnes nées d'un couple unissant un parent polonais à un immigré d'une autre origine et de $22 \%$ de ceux dont les deux parents sont des immigrants polonais. Le résultat rencontré par toutes les enquêtes démographiques consacrées à ce thème ${ }^{35}$, a pour nous d'importantes implications pratiques. Il nous faut en effet, si nous voulons raisonner sur les rapports unissant trajectoire, milieu de provenance et origine du conjoint, ne plus considérer que le sort des individus nés de deux immigrés polonais. Les autres groupes sont trop peu nombreux pour qu'une décomposition de ceux-ci selon des variables supplémentaires soit très pertinente et leur profil trop spécifique, origine des parents, milieu de provenance et trajectoire sociogéographique sont en effet corrélés, pour que les conserver n'aboutisse pas à brouiller nos données. Nous n'étudierons donc dans la section suivante de cet article que les enfants issus de deux immigrés d'origine polonaise.

\section{La classe ouvrière, conservatoire de la polonité}

L'étude du mariage de ces jeunes gens et de ces jeunes filles, aux comportements très similaires en ce domaine, puisque une même proportion de filles et de garçons

35 TRIBALAT M., De l'immigration à l'assimilation. Enquête sur les populations d'origine étrangère en France, Paris, La découverte/INED, 1995, page 101. 
épousent des personnes de même origine qu'eux, montre que le milieu sociogéographique d'origine pèse fortement sur le choix du conjoint.

Origine du conjoint selon le milieu sociogéographique de provenance

\begin{tabular}{|l|l|l|l|l|l|l|}
\hline Insertion sociogéographique des parents/Patronyme du conjoint & \multicolumn{2}{|c|}{ Polonais } & \multicolumn{2}{c|}{ Non polonais } & \multicolumn{2}{|c|}{ Total } \\
\hline & En \% & Effectifs & En \% & Effectifs & En \% & Effectifs \\
\hline Urbains installés & 87,8 & 43 & 12,2 & 6 & 100 & 49 \\
\hline Prolétaires vierzonnais & 82,2 & 37 & 17,8 & 8 & 100 & 45 \\
\hline Campagnards installés & 83,3 & 30 & 16,7 & 6 & 100 & 36 \\
\hline Ouvriers des campagnes agricoles & 85 & 164 & 15 & 29 & 100 & 193 \\
\hline Ouvriers des campagnes industrialisées & 80 & 24 & 20 & 6 & 100 & 30 \\
\hline Ouvriers des cités & 70,1 & 183 & 29,9 & 78 & 100 & 261 \\
\hline Total & 78,3 & 481 & 21,7 & 133 & 100 & 614 \\
\hline
\end{tabular}

Nous avons ici croisé l'origine $\mathrm{du}$ nom $\mathrm{du}$ conjoint et la situation sociogéographique des parents à la naissance des individus étudiés. Celle-ci est décrite par une variable synthétique, obtenue par classification postfactorielle ${ }^{36}$. L'opération consiste à dégager quelques types, puis à grouper autour d'eux les familles ressemblant le plus aux types définis. Nous pouvons alors distinguer des situations définies par un faisceau de variables, plus pertinentes que celles décrites par un unique facteur et évoquer les résultats obtenus de façon plus synthétique qu'en usant des seuls tris croisés, qui conduisent à multiplier les tableaux.

Nous avons distingué ici des urbains installés résidant, lorsqu'ils naissent, dans une ville du département comptant peu d'étrangers parmi ses habitants et dont le père occupe rarement un emploi de manoeuvre. Le prolétaire vierzonnais type est généralement né à Vierzon, d'un père manoeuvre, qui souvent n'a pas signé son acte de naissance. La majorité des prolétaires des campagnes agricoles sont nés de parents tous deux ouvriers agricoles et travaillant dans une campagne d'où les activités industrielles sont absentes. Le prolétaire des campagnes industrialisées conforme au type est né, au début de la période étudiée, dans l'est du département, où son père travaille comme manoeuvre ou journalier. Les pères des enfants des cités s'emploient, en tant que manoeuvres, dans une

36 Partition générée par un algorithme de classification mixte. Pour une présentation de cette technique voir LEBART L., MORINEAU A., PIRON M., Statistique exploratoire multidimensionelle, Paris, Dunod, 1995, pages 145 à 206. On se souviendra que ces techniques sont efficaces lorsqu'il s'agit d'explorer des données ou d'exposer des résultats mais que l'instabilité des partitions crées confère une faible valeur de preuve aux résultats ainsi obtenus qui doivent être confirmés en recourant à d'autres moyens. Les principales variables utilisées ici sont: l'année de naissance, la profession des deux parents, la taille de la localité de résidence, l'importance de l'activité industrielle dans la localité de résidence, la localisation géographique de la localité de résidence, les effectifs de la population étrangère présente dans la localité de résidence, la présence de la signature du père sur l'acte de naissance. 
usine de taille importante, située dans une commune à la population étrangère importante, leur mère est souvent inactive. Cela fait d'eux les seuls à naître en un lieu où la population polonaise est nombreuse. La classe des campagnards installés regroupe des enfants dont beaucoup ont un père agriculteur, installé en une campagne d'où les activités industrielles sont absentes.

Les enfants des cités sont de loin les plus nombreux à épouser un partenaire de même origine, ce que confirme une décomposition de nos données, $32 \%$ des enfants de Polonais dont les parents résident à Lunery-Rosières lors de leur naissance, de même que $28 \%$ de ceux provenant de Saint-Florent-sur-Cher, épousent un homme ou une femme de nom polonais, ce n'est le cas que de $9 \%$ de ceux venant de Bourges ${ }^{37}$. Nous rejoignons ainsi les conclusions de Girard et Stoetzel, qui avaient déjà mis en lumière le fait que la socialisation en un lieu où est présente une population de même origine nombreuse et structurée conduit plus fréquemment à prendre pour époux un individu de même origine. L'existence en effet d'institutions - fêtes collectives, associations permettant de multiplier les occasions de rencontrer des partenaires potentiels de même origine dépend étroitement de la taille de la population susceptible de soutenir ces institutions ${ }^{38}$. De plus, les marchés matrimoniaux étant, durant notre période, souvent strictement locaux et l'homogamie sociale étant la règle ${ }^{39}$, les partenaires potentiels des enfants des cités sont, pour une bonne part, de même origine qu'eux.

Il semble que les enfants des immigrés des villes soient plus nombreux à contracter un mariage qui les lie à un partenaire de même origine quand leur père appartient à la fraction la moins qualifiée de la classe ouvrière. Les enfants des ouvriers vierzonnais, pour la plupart fils de manoeuvres, sont en effet $18 \%$ à le faire, ce n'est le cas que de $12 \%$ des enfants des urbains installés. L'écart est faible cependant, vu la qualité de nos variables et les effectifs concernés. De plus, il est difficile de savoir ce qui revient à la position du père et ce qui renvoie au parcours des enfants eux-mêmes, puisque les fils et les filles des manoeuvres suivent plus souvent que les autres une trajectoire que nos données associent à de plus grandes chances de contracter mariage avec un partenaire de même origine.

Les formes de l'insertion socioprofessionnelle, autant que les caractéristiques du milieu de résidence des futurs conjoints, se révèlent en effet des facteurs déterminants.

\footnotetext{
${ }^{37}$ Les populations citées sont fortes respectivement de 200, 61 et 45 individus.

38 POINARD M., "Stratégies régionales et mouvement associatif: les Portugais en Aquitaine et Midi-Pyrénées", in, Collectif, Les Portugais en Aquitaine, des soutiers de l'Europe à l'esquisse d'un partenariat privilégié, Travaux et documents du CENPA, numéro 4, Éditions de la maison des sciences de l'homme d'Aquitaine, Bordeaux, 1990, pages 229-246.

${ }^{39}$ GIRARD A., Le choix du conjoint. Une enquête psycho-sociologique en France, Paris, PUF/Ined, Travaux et documents, Cahier numéro 44, 1964.
} 
L'établir suppose cependant de restreindre à nouveau les effectifs de notre population, puisque nous ne pouvons le faire qu'en prenant en compte les renseignements que nous fournissent les actes de mariage des enfants des Polonais du Cher.

Nous possédons 429 actes de ce type, 233 décrivent le mariage d'une fille, 198 celui d'un garçon né de deux immigrés polonais. Ces actes montrent que les enfants les plus susceptibles de s'unir à un conjoint de même origine sont les plus enracinés dans les lieux marqués avant guerre par une forte présence polonaise, tant parce qu'ils y résident, que parce que leurs parents y résident. Ainsi $31 \%$ des 145 enfants dont nous savons que leurs parents résident lors de leur mariage à Rosières ou à Saint-Florent-sur-Cher, localités ouvrières abritant avant guerre une population polonaise importante, épousent un conjoint portant un nom polonais, ce n'est le cas que de $17 \%$ des enfants dont les parents résident en dehors de ces localités. De même 29\% des 127 enfants résidant en ces lieux lors de leur mariage épousent un partenaire de même origine qu'eux. Nos données suggèrent de plus que ceux qui résident en ces lieux depuis leur enfance sont particulièrement nombreux à contracter de tels mariages, puisque c'est le cas de $31 \%$ d'entre eux, alors qu'une seule des 11 personnes les habitant lors de son mariage, mais n'y étant pas née, est en ce cas. Les effectifs étudiés sont cependant trop faibles pour que nous puissions l'établir avec certitude.

Les hommes et les femmes épousant des conjoints portant un nom polonais sont aussi les plus ancrés dans la condition ouvrière, voire la fraction la moins qualifiée de celle-ci; $24 \%$ des enfants dont le père se déclare manoeuvre lors de leur mariage nouent une telle union, ce qui est le cas de respectivement 19,13 et $12 \%$ des enfants d'ouvriers qualifiés, d'ouvriers agricoles et d'agriculteurs ${ }^{40}$. Nous ne faisons que retrouver là des résultats similaires à ceux obtenus plus haut, pour une population plus étendue. De plus, l'enracinement des parents dans les lieux de l'immigration est très étroitement corrélé à l'appartenance de ceux ci aux fractions les moins qualifiées de la classe ouvrière.

Ces deux facteurs pesant fortement sur le devenir social des enfants, nous constatons de même que ceux qui ont le plus de chance de contracter une union endogame sont souvent eux-mêmes ouvriers. Ainsi, si $25 \%$ des 88 filles se déclarant ouvrières lors de leur mariage sont en ce cas, nous ne trouvons parmi les 76 qui occupent un poste d'employée, ou appartiennent aux professions salariées qualifiées, que 8 partenaires de telles unions. De même, $26 \%$ des manoeuvres de sexe masculin sont en

${ }^{40}$ Les groupes distingués sont fort de respectivement, 158, 36, 23 et 58 individus. Les classifications utilisées dans la suite de ce texte suivent, le plus près qu'il est possible les prescriptions de l'ancien code Insee. La modestie de nos effectifs nous oblige cependant à des agrégations brutales. Ainsi, les contremaîtres sont ils rangés parmi les ouvriers qualifiés et regroupons nous parfois employés, cadres moyens et supérieurs en un même groupe de "'salariés non ouvriers". 
ce cas, ainsi que $22 \%$ des ouvriers qualifiés, mais seuls $15 \%$ des salariés non ouvriers de sexe masculin épousent une fille au patronyme polonais ${ }^{41}$.

L'ancrage dans la condition ouvrière des partenaires de conjoints de nom polonais a de plus de fortes chances d'être confirmé par leur mariage. Ils ont ainsi de grandes chances d'épouser des ouvriers ou des ouvrières.

Origine et Csp du conjoint des filles de deux immigrés polonais

\begin{tabular}{|c|c|c|c|}
\hline Csp conjoint/Patronyme du conjoint & Polonais & Non polonais & Total \\
\hline Agriculteurs & 2 & 11 & 13 \\
\hline$\%$ colonne & 4,3 & 5,9 & 5,6 \\
\hline$\%$ ligne & 15,4 & 84,6 & 100 \\
\hline \multirow[t]{3}{*}{ Patrons } & 1 & 12 & 13 \\
\hline & 2,2 & 6,5 & 5,6 \\
\hline & 7,7 & 92,3 & 100 \\
\hline \multirow[t]{3}{*}{ Employés et cadres } & 3 & 57 & 60 \\
\hline & 6,5 & 30,6 & 25,9 \\
\hline & 5,0 & 95,0 & 100 \\
\hline \multirow[t]{3}{*}{ Ouvriers qualifiés } & 19 & 73 & 92 \\
\hline & 41,3 & 39,2 & 39,7 \\
\hline & 20.7 & 79,3 & 100 \\
\hline \multirow[t]{3}{*}{ Manoeuvres } & 16 & 30 & 46 \\
\hline & 34,8 & 16,1 & 19,8 \\
\hline & 34,8 & 65,2 & 100 \\
\hline \multirow[t]{3}{*}{ Ouvriers agricoles } & 5 & 3 & 8 \\
\hline & 10,9 & 1,6 & 3,4 \\
\hline & 62,5 & 37,5 & 100 \\
\hline \multirow[t]{3}{*}{ Total } & 46 & 186 & 232 \\
\hline & 100 & 100 & 100 \\
\hline & 19,8 & 80,2 & 100 \\
\hline
\end{tabular}

Seules 6 des 46 filles qui épousent un homme portant un patronyme polonais, soit $13 \%$ d'entre elles, ne se marient pas avec un ouvrier. D'autre part, un "beau mariage", qui peut être pour les filles signe et/ou moyen d'une ascension sociale ${ }^{42}$, implique que l'on ne se marie pas avec un partenaire de même origine. Les filles épousant un salarié n'appartenant pas au monde ouvrier ne sont ainsi que 6,5\% à épouser un homme portant un nom à consonance polonaise. Si nous pouvons conclure que les couples unissant deux porteurs de noms polonais sont, pour la plupart, profondément ancrés dans la classe ouvrière locale, au moment du moins où ils se marient, ils ne s'en déduit cependant pas que la majorité des descendants de Polonais en ce cas contractent de telles unions. Ainsi, les deux tiers des filles d'immigrés épousant des manoeuvres ontelles pour partenaire un garçon ne portant pas un tel patronyme.

${ }^{41}$ Ces groupes rassemblent respectivement 39,98 et 40 individus.

${ }^{42}$ RYGIEL P., Mais où sont ..., opus cité, pages 639-642. 
Ces constats, que confirment l'examen de la profession des beaux-pères, des brus d'origine polonaise ${ }^{43}$, ou ceux des garçons d'origine polonaise ${ }^{44}$, s'expliquent en partie par la force des lois de l'homogamie sociale, dont on ne comprendrait pas qu'elles pèsent moins sur les mariages des enfants des Polonais que sur ceux de leurs contemporains ${ }^{45}$. Les ouvriers et les enfants d'ouvriers étant les plus nombreux à épouser des garçons et des filles de même origine, on ne saurait être surpris que leurs conjoints leur ressemblent et que l'on trouve parmi eux nombre de jeunes gens de même statut et de même origine. De plus, du fait de la structure socioprofessionnelle de la population d'origine polonaise, qui, dans le Cher du moins, est majoritairement ouvrière et d'origine ouvrière, les chances de trouver un partenaire de nom polonais, qui appartienne, par son métier et/ou son origine, aux classes moyennes urbaines, sont assez réduites. Ajoutons que les garçons et les filles d'origine polonaise qui s'allient à une famille appartenant aux classes moyennes salariées, et/ou épousent un salarié non ouvrier, sont souvent en ascension sociale ${ }^{46}$, trajectoire qui s'accompagne, pour la plupart d'entre eux, d'une sortie des lieux où s'installèrent les primo-migrants. Il leur faut alors, pour trouver un partenaire proche d'eux, qui soit de même origine et de même statut qu'eux, beaucoup de chance, ou de persévérance. Enfin, de telles trajectoires sont souvent le fait des derniers nés des enfants des Polonais du Cher, alors que les plus âgés sont plus nombreux à reproduire le statut des parents, comme à épouser un conjoint de même origine qu'eux.

\section{Le choix du conjoint, révélateur des contours d'un espace de relations} sociales.

Il nous reste à déterminer la signification de nos données, et d'abord à nous demander si épouser un conjoint de nom polonais est le signe que l'on se meut au sein d'un espace de relation structuré, au moins en partie, par l'origine des parents. Nous pouvons le vérifier en examinant si épouser un individu portant un nom polonais conduit fréquemment à choisir comme témoin des personnes qui partagent cette origine.

$4371 \%$ de celles qui épousent un garçon de nom polonais ont pour beau-père un ouvrier et $5 \%$ de celles dont le beau père se déclare lors de leur mariage employé ou cadre épousent un garçon de nom polonais. La population de référence est ici constituée des filles nées de deux immigrés polonais dont l'acte de mariage indique la profession du beau-père, elles sont 150 .

$4470 \%$ de ceux qui épousent une fille de nom polonais ont pour beau père un ouvrier et $6 \%$ de ceux ayant un beau père employé ou cadre épousent une fille de nom polonais. Nous connaissons la profession de 152 beaux pères, 33 de ceux-ci ont pour gendre le fils de deux immigrés polonais.

45 GIRARD A., Le choix du conjoint. Une enquête psycho-sociologique en France, Paris, PUF/Ined, Travaux et documents, Cahier numéro 44, 1964.

${ }^{46}$ RYGIEL P., Mais où sont ..., opus cité, pages 639-642. 
Origine des noms du conjoint et des témoins des enfants de Polonais

\begin{tabular}{|c|c|c|c|}
\hline Nombre de témoins de nom pol/Nom du conjoint & Polonais & Non polonais & Total \\
\hline Aucun & 18 & 250 & 268 \\
\hline$\%$ colonne & 18,0 & 56,7 & 49,5 \\
\hline$\%$ ligne & 6,7 & 93,3 & 100 \\
\hline \multirow[t]{3}{*}{$\mathrm{Un}$} & 37 & 169 & 206 \\
\hline & 37,0 & 38,3 & 38,1 \\
\hline & 18,0 & 82,0 & 100 \\
\hline \multirow[t]{3}{*}{ Deux } & 45 & 22 & 67 \\
\hline & 45,0 & 5,0 & 12,4 \\
\hline & 67,2 & 32,8 & 100 \\
\hline \multirow[t]{3}{*}{ Total } & 100 & 441 & 541 \\
\hline & 100 & 100 & 100 \\
\hline & 18,5 & 81,5 & 100 \\
\hline
\end{tabular}

Ce tableau témoigne à la fois de la force des liens et des réseaux nés d'une origine polonaise, puisque plus de la moitié des mariés ont au moins un témoin portant un nom polonais et de ce que le fait d'épouser un partenaire de nom polonais et d'avoir parmi ses témoins des personnes possédant un tel nom sont liés, ce qui exprime, au moins en partie, la participation des individus en ce cas à un espace de relations où les personnes d'origine polonaise sont particulièrement nombreuses.

D'autres éléments peuvent nourrir ce constat. D'une part les enfants d'immigrés dont un frère ou une soeur a épousé un partenaire de nom patronymique polonais sont beaucoup plus enclins à contracter une union du même type, c'est le cas de 30\% des 114 personnes en ce cas, alors que seuls $16 \%$ des 214 individus ayant des frères ou soeurs dont les actes de naissance ne mentionnent que des mariages avec des partenaires sans nom polonais épousent un homme ou une femme de nom polonais.

De même, si nous restreignons notre analyse aux enfants de Polonais dont l'acte de mariage d'un des frères, ou de l'une des soeurs, nous est connu, nous constatons que $63 \%$ de ceux qui ont épousé un individu de même ascendance qu'eux ont un frère ou une soeur dont l'un au moins des témoins portait un patronyme polonais, alors que ce n'est vrai que de $45 \%$ de ceux dont rien ne laisse supposer que leur partenaire est d'origine polonaise ${ }^{47}$.

Les hommes et les femmes dont les premiers conjoints portent un nom de même origine que le leur ont donc, plus souvent qu'à leur tour, des beaux-frères et des bellessoeurs, des témoins, partageant cette origine, voire des frères et des soeurs dont les témoins portent eux aussi un nom polonais, ce qui laisse supposer que l'assimilation est affaire de famille plus que d'individus.

L'étude des mariages de ces enfants de Polonais - révélateurs efficaces des réseaux de relations auxquels ils appartiennent - témoigne de la force de ce que d'autres ont

${ }^{47}$ Populations de référence fortes de respectivement 63 et 47 individus. 
nommé le "lien communautaire", que manifeste ici la fréquence du recours à des témoins dont nous pouvons supposer qu'ils sont d'origine polonaise. Même si cependant une notable partie des enfants des Polonais participent à des réseaux que structure manifestement l'origine, l'assimilation est en marche, elle apparaît même particulièrement rapide et brutale. La réorientation des stratégies familiales lorsque l'espoir du retour s'estompe, la diversification des parcours, qui conduit à une dispersion géographique des populations étudiées, autant qu'à leur diversification sociale, l'accélère, prodigieusement; les membres des générations nées dans les années trente sont deux fois moins nombreux que leurs aînés à contracter mariage au sein du groupe. Nous en tirons deux enseignements. D'une part, nous sous-estimons peut-être la rapidité avec laquelle les migrations anciennes se fondirent dans la population de la France, parce que leur étude est souvent menée à partir des lieux ou elle donnèrent naissance à des populations nombreuses et regroupées, lieux qui s'avèrent ici des conservatoires efficaces de la polonité, mais dont l'observation conduit à écrire une histoire fort différente de celle qu'autorise la prise en compte du destin de ceux qui les quittèrent rapidement, voire n'y vécurent pas. Le fait que souvent les histoires des populations immigrées de l'entredeux-guerres s'arrêtent à la veille, ou à la fin, de la seconde guerre mondiale, a les mêmes effets. Il s'ensuit que, raisonnant sur l'assimilation de ces populations, nous évoquons peut être des groupes dont les membres, nés et socialisés en France, y ont aussi contracté des unions qui font rentrer une majorité d'entre eux au sein de familles ne partageant pas leur origine, ce qui demanderait cependant pour être établi des enquêtes menées dans un cadre plus large. La généralisation des conclusions obtenues se heurte en effet à de puissants obstacles.

De fait, ce travail a pour cadre un département dont les structures économiques et sociales ne sont similaires, ni à celles dessinées par une hypothétique moyenne nationale, ni à celles des grandes régions d'immigration. Le secteur industriel y est marqué par l'opposition entre le secteur privé et un secteur d'état puissant et attractif, pour l'essentiel concentré à Bourges. Cela peut expliquer que le parcours de peu d'enfants d'immigrés se déroule sur les lieux qui avaient vu s'installer leurs familles. D'autre part, le Cher se caractérise par la faiblesse persistante des emplois tertiaires. Il est donc possible qu'ici plus qu'ailleurs la conquête d'un diplôme contraigne à quitter la région de son enfance, d'autant plus que Paris est proche, et donc, pour les enfants des Polonais de Rosières, la communauté au sein de laquelle ils ont grandi. De plus, la classe ouvrière du Cher, à laquelle s'intègre la majorité des enfants de polonais demeurés sur place, a ses spécificités, au nombre desquelles l'ancienneté et la puissance de ses organisations et de ses institutions, qui contribuent à structurer puissamment les modes de sociabilité 
locaux ${ }^{48}$ et sont très présentes, dès le début de la période, au sein des écosystèmes ouvriers rejoints par ces populations, écosystèmes dont la formation est largement antérieure à leur arrivée. Il est permis de penser, même si nous aurions besoin d'éléments de comparaison pour l'établir, que cela n'est pas sans effets sur les formes de l'intégration sociale, et donc des comportements matrimoniaux, des populations issues de l'immigration.

Cette étude illustre enfin la puissance des mécanismes sociaux et des effets de contexte, qui pèsent fortement sur celles des pratiques sociales qui engagent le plus l'identité des individus. Que les communautés homogènes formées durant l'entre-deuxguerres se disloquent, que les individus les quittent et tant la fréquence des mariages engageant deux conjoints de même origine, que les chances, pour l'individu, de contracter un tel mariage, s'effondrent, même lorsque nous avons affaire aux enfants de ceux que les observateurs sociaux ont longtemps jugés les plus inassimilables de tous les immigrés présents en France, au point que Philippe Ariès pouvait encore écrire en 1948 que :

" [ces] étrangers inassimilables (...) vivent en groupes fermés, avec leurs églises, leurs écoles, leurs magasins, leurs jeux, étrangers au reste de la population ${ }^{49 "}$.

L'ampleur des variations enregistrées, d'une cohorte à l'autre, d'un lieu à l'autre, conduit à douter que la référence à la culture des parents puisse beaucoup aider à comprendre les formes de l'intégration sociale des enfants d'immigrés. Les enfants d'origine polonaise n'existent pas en tant que groupe homogène au regard des pratiques étudiées, non plus d'ailleurs, malgré l'évidente visibilité sociale des populations regroupées près des cités usines, en tant que groupe socio-ethnique homogène, mais sont le produit de la réunion de populations disjointes, dont les membres ont des pratiques fortement structurées par la trajectoire de leur famille et la leur propre. Il s'ensuit d'une part que tout taux caractérisant le groupe n'est qu'une moyenne masquant de profonds écarts, soit un artefact statistique. D'autre part, le constat de l'existence, localement, d'une segmentation socio-ethnique ne saurait garantir la pertinence de l'usage de catégories fondée sur l'origine dans un cadre plus large, i.e. celui de la région ou de la nation, du moins si le recours à celles-ci est censé avoir une valeur explicative. Le constat est ancien ${ }^{50}$, à l'heure où l'opportunité d'un recours à des classifications

48 PIGENET M., Les ouvriers du Cher fin XVIII -1914, Travail espace et conscience sociale, IHS-CCEES CGT, 1990, PIGENET (M. et M.), RYGIEL R., PICARD M., Terre de luttes, les précurseurs (1848-1939), Éditions sociales, Paris, 1977.

49 ARIES P., Histoire des populations françaises, Paris, Le Seuil 1971, pages 110-111. Première édition 1948.

${ }^{50}$ NOIRIEL G., Le creuset français, Paris, Seuil, 1988. 
ethniques est en débat ${ }^{51}$, il est sans doute bon de le répéter. Il conduit en effet à douter singulièrement de l'utilité de l'entrée de telles variables dans l'arsenal permanent de l'appareil statistique d'État. Du fait des spécialisations sectorielles et géographiques fortes qui caractérisent chaque immigration ${ }^{52}$ et du lien très fort entre le mode d'insertion des populations immigrées et le devenir ${ }^{53}$ et les choix des enfants issus d'elles, toute classification fondée sur l'origine enregistrera nécessairement des écarts importants au niveau macrosociologique, permettant d'opposer les sous groupes qu'elle distinguera, en particulier lorsque sera étudiée leur assimilation, puisque l'origine fonctionnera comme un marqueur social, signalant des formes spécifiques d'insertion sociogéographique, fortement corrélées aux indices décrivant l'assimilation des populations observées. De plus, il est, pour les mêmes raisons, et en dehors d'un cadre strictement local, illusoire de vouloir comparer l'assimilation de deux populations ainsi définies toutes choses égales par ailleurs, c'est à dire en faisant abstraction des formes de l'insertion de leurs membres dans la société française. La valeur heuristique d'une telle variable explicative risque fort donc de se révéler faible. Souvent redondante, elle dérogera au principe d'économie qui régit l'activité interprétative ${ }^{54}$. Les effets politiques de l'adoption d'un tel mode de classement social seront moins bénins, puisque seront mis en circulation, revêtus du sceau de l'État, des données dont le mode de construction même permettra une hiérarchisation des groupes distingués.

51 MERLLIE D., SPIRRE A., "La question des origines dans les statistiques en France. Les enjeux d'une controverse", Le mouvement social, juillet-septembre 1999, numéro 188.

52 Pour un exemple précis concernant les migrations de l'Entre-deux-guerres voir, GUICHARD E., LE GUILLOU O., MANITAKIS N., NOIRIEL G., "Les étrangers et les naturalisés dans la société française. Commentaire des recensements de 1931 et 1936", GUICHARD E., NOIRIEL G., Construction des nationalités, opus cité.

${ }^{53}$ RYGIEL P., Mais où sont..., opus cité.

54 ECO U., Les limites de l'interprétation, Paris, Grasset, première édition 1990, édition française 1992, pages 369-384. 


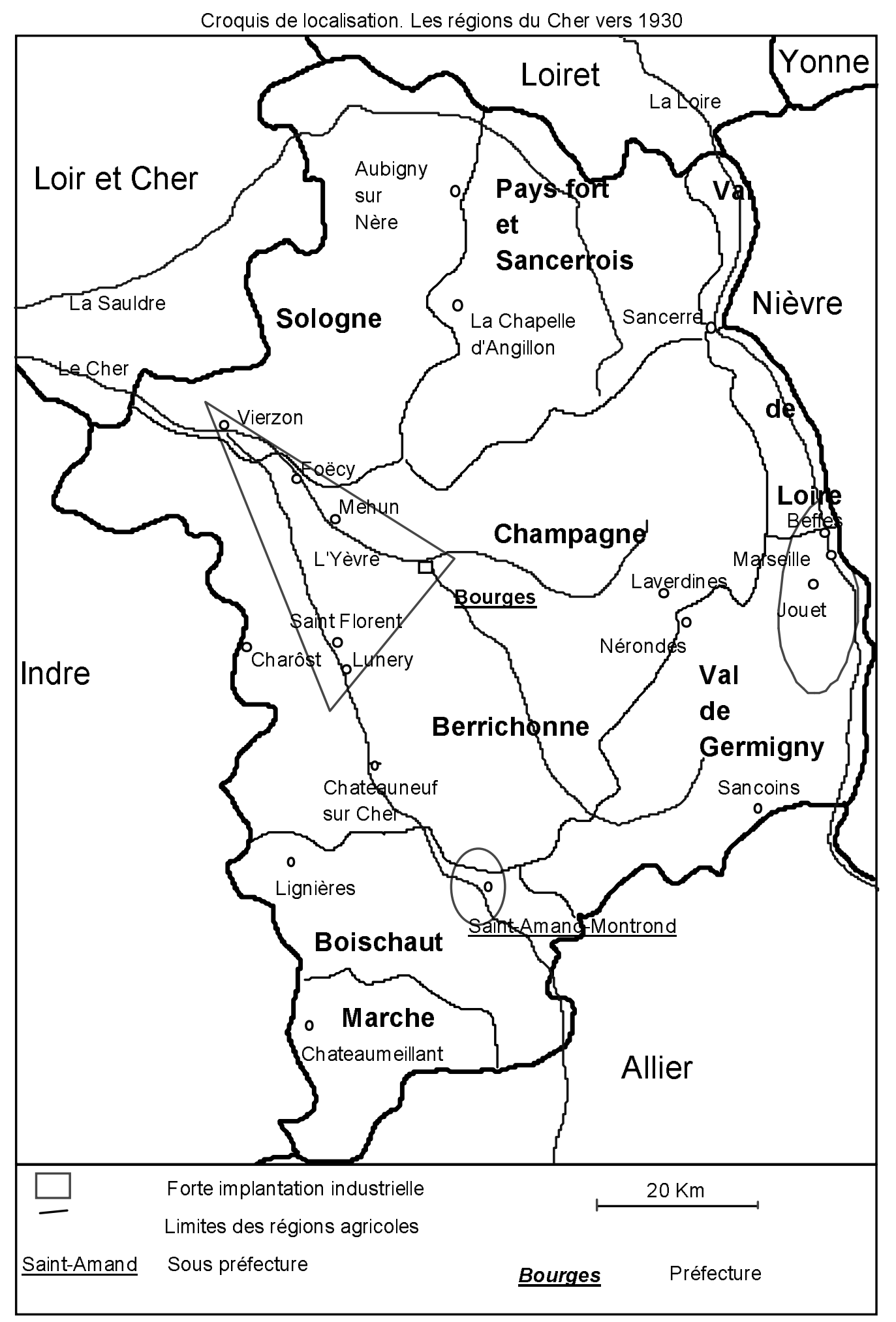




\title{
Résumé
}

Les enfants des familles polonaises implantées dans le Cher entre 1920 et 1945 sont peu nombreux à épouser des enfants de Polonais. De plus, la probabilité qu'ils contractent un tel mariage dépend très fortement de la génération à laquelle ils appartiennent, mais aussi de la trajectoire sociale et géographique de leur famille et de la leur propre. Les plus enclins à le faire sont les plus âgés d'entre eux et les plus ancrés dans la condition ouvrière et les lieux marqués avant la seconde guerre mondiale par une forte immigration. L'ampleur des écarts enregistrés permet de conclure que le groupe étudié n'est pas homogène au regard des pratiques étudiées et que le taux de mariage mixte, souvent pris comme critère de l'assimilation sociale d'une population, est fortement lié aux formes de l'insertion sociale et géographique de celle-ci et aux transformations du contexte historique. Nous pouvons alors douter de l'efficacité d'une explication des formes de l'insertion sociale d'une population issue de l'immigration qui ferait de l'origine et de la culture des parents le pivot de son interprétation, autant que de la nécessité de l'introduction dans l'arsenal statistique de l'État de classements sociaux fondés sur l'origine nationale des individus, qui souvent postulent l'homogénéité de ces groupes.

\begin{abstract}
Few of the children of Polish-born parents who settled in the Cher region during the first half of the twentieth century married people of Polish background. The likelihood of such a marriage depended on the date of birth, geographical location, social status and parents status of each individual. The number of marriages within the group was highest amongst the oldest children, the one who, the most clearly, belonged to the working class and lived in the places that had, prior to WWII, housed large immigrant communities. The rate of intermariage, often regarded as the ultimate criterion of assimilation, is then very strongly linked to the social and geographical conditions in which the members of this population lived, as well as to the changes that occurred in the historical context. Therefore, we can not explain the way a population assimilates solely in reference to the origin or the culture of the immigrants who gave birth to it and can question the need for the introduction of ethnic categories in the french census.
\end{abstract}

